Agricultura familiar no norte do estado do Rio de Janeiro: identificação de manejo tradicional

Raquel da Silva Paes, Camilah Antunes Zappes

\title{
AGRICULTURA FAMILIAR NO NORTE DO ESTADO DO RIO DE JANEIRO: IDENTIFICAÇÃO DE MANEJO TRADICIONAL
}

\section{Family farm in north of Rio de Janeiro state: identification of traditional management}

Raquel da Silva Paes

Universidade Federal Fluminense, Campos dos Goytacazes, Rio de Janeiro, Brasil. raquelspaes@hotmail.com

Camilah Antunes Zappes

Universidade Federal Fluminense, Campos dos Goytacazes, Rio de Janeiro, Brasil. camilahaz@yahoo.com.br

Artigo recebido em 26/05/2016 e aceito para publicação em 29/11/2016

RESUMO: O objetivo deste estudo é descrever as técnicas utilizadas na agricultura familiar e identificar a ocorrência de um possível manejo tradicional na atividade praticada no $5^{\circ}$ Distrito do município de São João da Barra, estado do Rio de Janeiro. Para a coleta das informações foram realizadas 50 entrevistas etnográficas com agricultores familiares por meio de questionários semiestruturados contendo perguntas abertas e fechadas, observação participante e diário de campo. Os agricultores são em sua maioria do sexo masculino com baixa escolaridade. $\mathrm{O}$ conhecimento tradicional sobre a agricultura é ensinado principalmente dentro do núcleo familiar. Para a agricultura familiar podem ser utilizadas ferramentas manuais e maquinário pesado. Para incremento de nutrientes no solo são utilizadas adubação química e orgânica e lodo de cana de açúcar. A irrigação ocorre por meio de poços artesianos/bomba elétrica/mangueira pressurizada e aspersão convencional. Para descanso do solo e melhor produção das lavouras são aplicadas rotação de culturas e pousio de áreas sendo estas caracterizadas como manejo tradicional na região.

Palavras-chave: Conhecimento tradicional; uso do solo; pequeno agricultor; norte do Rio de Janeiro.

ABSTRACT: The aim of this study is describe the techniques used in family farming and to identify the occurrence of a possible traditional management in the activity practiced in $5^{\circ}$ District of São João da Barra, State of Rio de Janeiro. For the collection of information were carried 50 interviews ethnographic with family farmers through semi structured questionnaires with open and closed questions, participant observation and field diary. Farmers are mostly males with low education. The traditional knowledge of agriculture is taught mainly within the nucleus of family. For the family farm can be used hand tools and heavy machinery. To increment of nutrient in the soil are used fertilizing chemistry and organic and slime of sugarcane. The irrigation occurs by use of artesian wells/electric pump/pressurized hose and sprinkler. For soil rest and better cultivation of crop yields are applied crop rotation and fallow areas which were characterized the traditional management in the region.

Keywords: traditional knowledge; land use; small farmer; north of Rio de Janeiro. 
Agricultura familiar no norte do estado do Rio de Janeiro: identificação de manejo tradicional

Raquel da Silva Paes, Camilah Antunes Zappes

\section{INTRODUÇÃO}

Agricultura familiar é caracterizada como uma atividade rural cujo trabalho apresenta relações de parentesco em que a gestão é realizada pelos proprietários das unidades produtivas, o capital pertence à família e os seus membros residem nas propriedades rurais (ABRAMOVAY, 1997; WANDERLEY, 1999; ALTAFIN, 2007). Tal atividade influencia decisivamente nas políticas públicas ligadas ao setor já que a base é de cunho familiar tradicional sendo regulamentada pela Lei Federal no 11.326/2006.

$\mathrm{Na}$ agricultura familiar são utilizadas técnicas específicas desenvolvidas a partir da prática diária da atividade com conhecimento adquirido durante anos pela comunidade local (ABRAMOVAY, 1997). Ainda, a relação dos agricultores familiares com a natureza interfere nas estratégias de manuseio e utilização dos recursos durante os cultivos agrícolas e nos ambientes onde são cultivados (POSEY, 1987). Por utilizarem técnicas locais, os agricultores familiares podem desenvolver manejo tradicional por meio do uso do próprio conhecimento tradicional (NETTING, 1993).

Manejo é compreendido como implementações de políticas públicas a fim de monitorar áreas naturais e as relações dos elementos sistêmicos para sustentar as funcionalidades dos ecossistemas (HANAZAKI, 2003). No manejo tradicional ocorre a integração entre atividades de comunidades locais que levam à conservação da natureza em ambientes intensamente utilizados pelos sistemas agrícolas, florestais e agroflorestais principalmente (CHRISTENSEN et al., 1996). Em todo o mundo há registros de domesticação e manejo de espécies vegetais por comunidades locais em que ao longo dos anos foram desenvolvidas técnicas de manejo tradicional (NETTING, 1993; PECH et al., 2004; ALTIERI; TOLEDO, 2005). O manejo tradicional tem sido atribuído como um importante elemento a ser considerado na política de gestão dos recursos naturais (GELCICH et al., 2006). Isso porque as experiências ancestrais de comunidades locais com a natureza podem contribuir na implementação e manutenção de políticas co-participativas de usos dos recursos da natureza (BERKES et al., 1998; SOUZA, 2006).

No norte do estado do Rio de Janeiro, especificamente no município de São João da Barra, a agri- cultura familiar apresenta práticas tradicionais em que os cultivos são desenvolvidos por meio de adubação orgânica e química com práticas de conservação dos solos que envolve rotação dos plantios e descanso do terreno pelo pousio (BURLA et al., 2015). Nesta região está inserido o $5^{\circ}$ Distrito do município de São João da Barra, onde em 2008 foram iniciadas as obras de instalação do Complexo Logístico Industrial Portuário do Açu (CLIPA) e suas atividades portuárias inauguradas em 2015. A instalação desse megaempreendimento pode ter interferido negativamente na agricultura familiar regional, pois houve uma intensificação da salinidade do solo provavelmente devido à construção do canal de atracação dos navios (PIRES, 2009; SOUZA, 2010). Tal interferência pode ter causado alteração nas práticas da agricultura familiar na região bem como ter induzido o surgimento de um manejo tradicional na atividade.

A identificação da prática de manejo tradicional em comunidades agrícolas apresenta importância a partir do momento em que podem ser encontradas soluções para problemas ambientais baseadas em técnicas locais de baixo custo e impacto para o ecossistema. Nesse sentido, esta pesquisa tem como objetivo descrever técnicas utilizadas na agricultura familiar praticada no $5^{\circ}$ Distrito do município de São João da Barra, norte do estado do Rio de Janeiro, além de identificar a ocorrência de um possível manejo tradicional voltado para a atividade agrícola na região.

\section{MATERIAL E MÉTODOS}

\section{Area de estudo}

No norte do estado do Rio de Janeiro está localizado o $5^{\circ}$ Distrito do município de São João da Barra com distância aproximada de $30 \mathrm{~km}$ da sede municipal (21 ${ }^{\circ} 52^{\prime}$ 'S e $040^{\circ} 55^{\prime} \mathrm{O}$ ) (IBGE, 2015). Neste distrito estão inseridas as localidades da Barra do Açu, Mato Escuro, Água Preta, Campo da Praia, Campo de Areia, Pipeiras, Barra do Jacaré, Cazumbá e Sabonete (Figura 1). Estas são identificadas como comunidades tradicionais por desenvolverem atividades de maneira ancestral que se dividem em pesca artesanal, artesanato tradicional e principalmente agricultura familiar (SOUZA et al., 2009; ECOLOGUS, 2011; BURLA et

Soc. \& Nat., Uberlândia, 28 (3): 385-395, set/dez/2016 
Agricultura familiar no norte do estado do Rio de Janeiro: identificação de manejo tradicional Raquel da Silva Paes, Camilah Antunes Zappes

al., 2015). Nesta região foi instalado o Complexo Logístico Industrial Portuário do Açu (CLIPA) (2149'S e $\left.041^{\circ} 00^{\prime} \mathrm{O}\right)$ onde estão inseridos a Zona Industrial do Porto do Açu (ZIPA) e o Distrito Industrial de São João da Barra (DISJB) com área próxima de 7.036 ha (ECOLOGUS, 2011).

Figura 1 - Indicação das comunidades estudadas no município de São João da Barra, norte do estado do Rio de Janeiro.

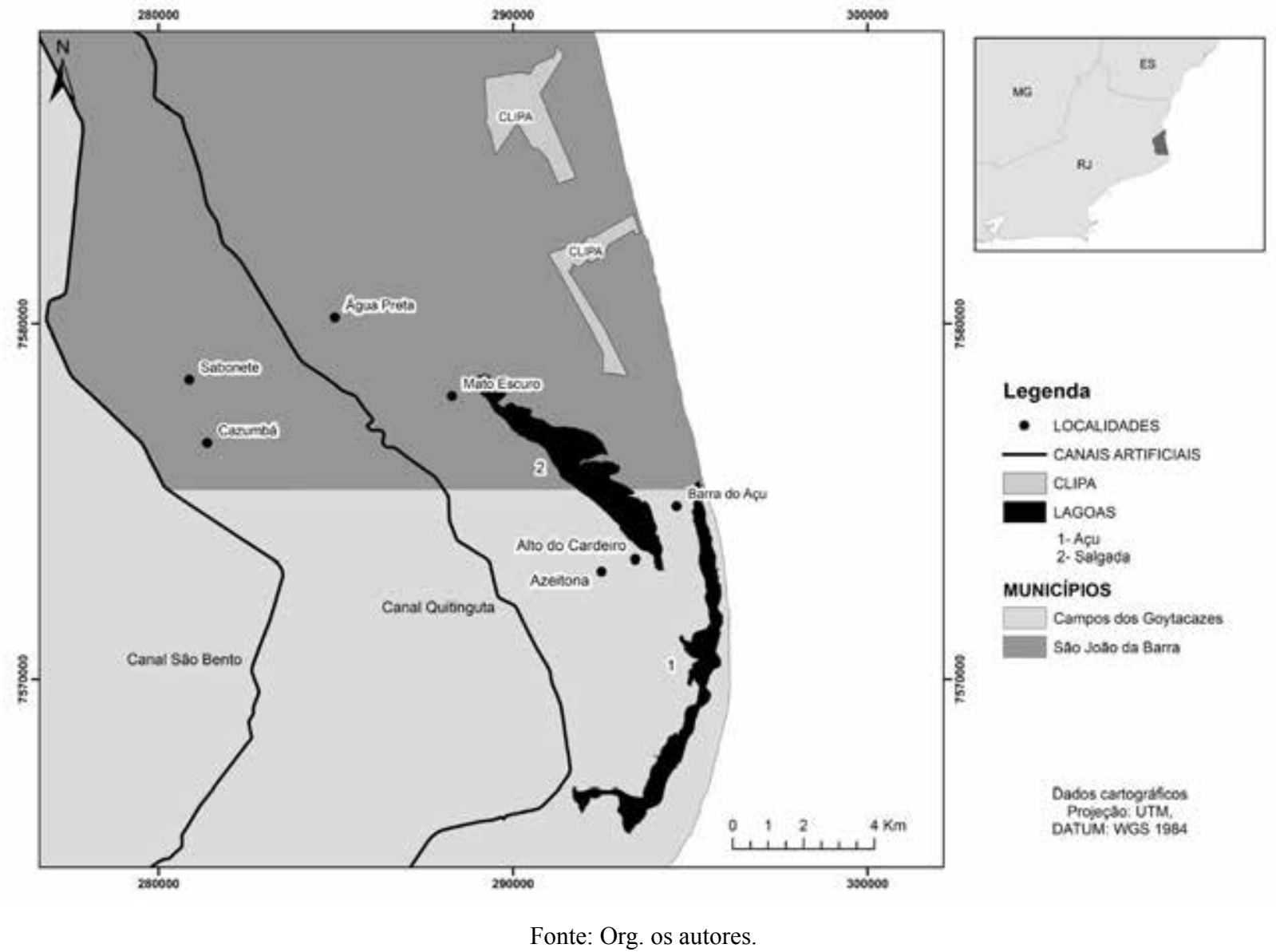

\section{Procedimentos}

Entre os meses de junho e outubro de 2015 foram realizadas 50 entrevistas etnográficas com agricultores familiares nas comunidades de Cazumbá $(\mathrm{n}=1)$, Sabonete $(\mathrm{n}=18)$, Mato Escuro $(\mathrm{n}=5)$, Barra do Açu ( $(n=3)$, Alto do Cardeiro $(n=6)$, Azeitona $(n=8)$ e Água Preta ( $\mathrm{n}=9)$. A fim de minimizar a interferência de outro entrevistado cada entrevista foi conduzida individualmente. $\mathrm{O}$ número de entrevistas realizadas $(n=50)$ é justificado: 1$)$ a partir da décima entrevista há um padrão de respostas entre os entrevistados em estudos etnológicos, e 2) trabalhos relacionados a megaempreendimentos e comunidades locais utilizaram números amostrais semelhantes (COUTINHO, 2009; PIRES, 2009; SOUZA, 2010).

Na primeira fase do trabalho foi realizada a observação participante a fim de acompanhar a prática agrícola (preparação do solo e sementes, técnicas de plantio e irrigação, colheita e venda dos produtos) e o cotidiano dos agricultores locais (MALINOWSKI, 1978). Neste momento também foi utilizado um diário de campo para registro de informações pertinentes à pesquisa (ALBUQUERQUE et al., 2010). A seleção do primeiro entrevistado ocorreu através de um guia local, já que geralmente é aquele que melhor conhece os atores locais (SANCHES, 2004), a partir do segundo entrevistado foi utilizada a técnica bola-de-neve 
Agricultura familiar no norte do estado do Rio de Janeiro: identificação de manejo tradicional

Raquel da Silva Paes, Camilah Antunes Zappes

(BAILEY, 1982), além de encontros aleatórios oportunísticos com agricultores.

Expressões e palavras locais foram utilizadas durante as entrevistas a fim de gerar maior confiança entre as partes com aproximação da linguagem local (COSTA-NETO \& MARQUES, 2010). As entrevistas foram guiadas por um questionário-padrão contendo questões abertas e fechadas semi-estruturadas exibindo certa flexibilidade e permitindo que o entrevistador faça as adaptações necessárias (KENDALL, 2008; ALBUQUERQUE et al., 2010). O questionário continha perguntas relacionadas ao perfil social dos entrevistados (sexo, idade e escolaridade), características da agricultura familiar local, modos de reprodução do conhecimento sobre a atividade, representações simbólicas, espécies cultivadas, tempo de atuação na prática da agricultura familiar e equipamentos e técnicas utilizadas na atividade.

Os relatos foram organizados em categorias de acordo com o questionário (RYAN \& BERNARD, 2000). A partir dessas informações foi possível descrever o conhecimento tradicional sobre a agricultura familiar, as técnicas tradicionais utilizadas e identificar possíveis alterações técnicas da atividade relacionadas a um possível manejo tradicional. Essas comparações foram realizadas a partir das frequências percentuais das respostas dos questionários.

\section{RESULTADOS}

\section{Perfil dos entrevistados e caracterização de técnicas agrícolas locais}

Dentre os entrevistados, $76 \%(\mathrm{n}=38)$ era do sexo masculino e $24 \%(n=12)$ do sexo feminino. A faixa etária variou entre 20 e 75 anos de idade, na qual: $16 \%(n=8)$ entre 20 e 30 anos; $10 \%(n=5)$ entre 31 e 40 anos; $26 \%(n=13)$ entre 41 e 50 anos; $30 \%(n=15)$ entre 51 e 60 anos; $12 \%(n=6)$ entre 61 e 70 anos e $6 \%$ $(n=3)$ entre 71 e 75 anos. Em relação à escolaridade $78 \%(\mathrm{n}=39)$ cursou o ensino fundamental, na maioria das vezes incompleto; $8 \%(n=4)$ o ensino médio; $8 \%$ $(n=4)$ não estudou e $6 \%(n=3)$ não respondeu.

A agricultura familiar praticada nas localidades do $5^{\circ}$ Distrito do município de São João da Barra, utiliza técnicas, instrumentos e etapas de plantio com baixo uso de tecnologia em que o conhecimento sobre a atividade é repassado oralmente no interior familiar. Segundo os agricultores, a reprodução ancestral do conhecimento envolve os ensinamentos do pai $(\mathrm{n}=16$; $32 \%$ ) e da família ( $\mathrm{n}=29 ; 58 \%$ ), mas também pode envolver o aprendizado por observação em que o agricultor aprende sozinho $(n=3 ; 6 \%)$, além do aprendizado ensinado pelos patrões $(n=2 ; 4 \%)$.

Ainda, foram relatadas as representações simbólicas relacionadas às propriedades rurais em que para $86 \%(\mathrm{n}=43)$ dos agricultores as propriedades rurais são patrimônio da família, para $68 \%(\mathrm{n}=34)$ a propriedade é uma ferramenta de trabalho e para $4 \%(n=2)$ não há simbolismo relacionado à terra. A diferença entre o número de entrevistados $(\mathrm{n}=50)$ e o número de respostas $(\mathrm{n}=79)$ é explicado devido alguns agricultores atribuírem mais de um significado em relação às propriedades.

As espécies cultivadas envolvem mandioca $(\mathrm{n}=10 ; 20 \%)$, batata doce $(\mathrm{n}=12 ; 24 \%)$, maxixe $(\mathrm{n}=21$; $42 \%)$, abacaxi $(n=25 ; 50 \%)$ e quiabo $(n=33 ; 66 \%)$. Outras espécies são cultivadas em pequena escala $(n=34 ; 68 \%)$ como melancia, laranja, pepino, salsinha, cebolinha e couve. Segundo os agricultores estas foram as espécies selecionadas ao longo dos anos, pois se adaptaram às características naturais da região costeira do norte fluminense, que apresenta condições de alta luminosidade e temperaturas, e solos com baixa fertilidade. O número de respostas sobre as espécies cultivadas ( $\mathrm{n}=135)$ é maior que o número de entrevistados $(\mathrm{n}=50)$, pois um mesmo agricultor trabalha com mais de um tipo de cultivo. Em relação ao tempo de prática na agricultura familiar na região, os agricultores atuam na atividade: até 15 anos ( $\mathrm{n}=18 ; 36 \%)$; de 16 a 30 anos $(\mathrm{n}=14 ; 28 \%)$; de 31 a 45 anos (n=9; 18\%); de 46 a 60 anos $(\mathrm{n}=8 ; 16 \%)$ e acima de 60 anos $(\mathrm{n}=1 ; 2 \%)$.

Para a realização da agricultura familiar alguns agricultores ( $\mathrm{n}=43)$ utilizam equipamentos e técnicas modernas como trator $(n=34 ; 68 \%$ ) (Figura $2 A)$ e o uso de adubos químicos ( $\mathrm{n}=9 ; 18 \%$ ) (Figura 2B) a fim de facilitar a preparação do solo, melhorar a fertilidade do mesmo para plantio e evitar pragas. Ainda, todos os agricultores utilizam ferramentas manuais como arados e enxadas. $\mathrm{O}$ uso de trator pode ser viabilizado aos agricultores por meio de auxílio público em que a prefeitura disponibiliza o maquinário via projeto social denominado 'Patrulha Mecanizada' ou pelo 
Agricultura familiar no norte do estado do Rio de Janeiro: identificação de manejo tradicional

Raquel da Silva Paes, Camilah Antunes Zappes

uso de maquinário disponibilizado pela associação de agricultores da região. Caso a demanda por estes maquinários seja intensa alguns agricultores precisam alugar outro trator a fim de não terem prejuízo com a lavoura. Em relação à irrigação agrícola, $100 \%(\mathrm{n}=50)$ dos agricultores descreveram utilizar poços artesianos, destes $84 \%(n=42)$ irrigam com o auxílio de bomba elétrica e mangueira pressurizada (Figura 2C) e 16\% $(n=8)$ afirmaram irrigar as lavouras por meio de poços artesianos via modelo aspersão convencional (Figura 2D). Nesta técnica de irrigação são fixados sistemas de canos na área central da lavoura em que são liberados jatos de água semelhantes a gotas de chuva. Um agricultor descreveu que em lavouras de abacaxi é utilizada uma técnica de proteção da fruta em que esta é envolta em folhas de jornal para evitar queimaduras em seu estágio final de desenvolvimento devido à luminosidade solar.

Figura 2 - Técnicas e instrumentos modernos utilizados na agricultura familiar no município de São João da Barra, norte do estado do Rio de Janeiro. A - Utilização de trator para preparação do solo; B - Aplicação de fertilizantes; C - Poço artesiano, bomba elétrica e magueira pressurizada; D - Aspersão convencional.
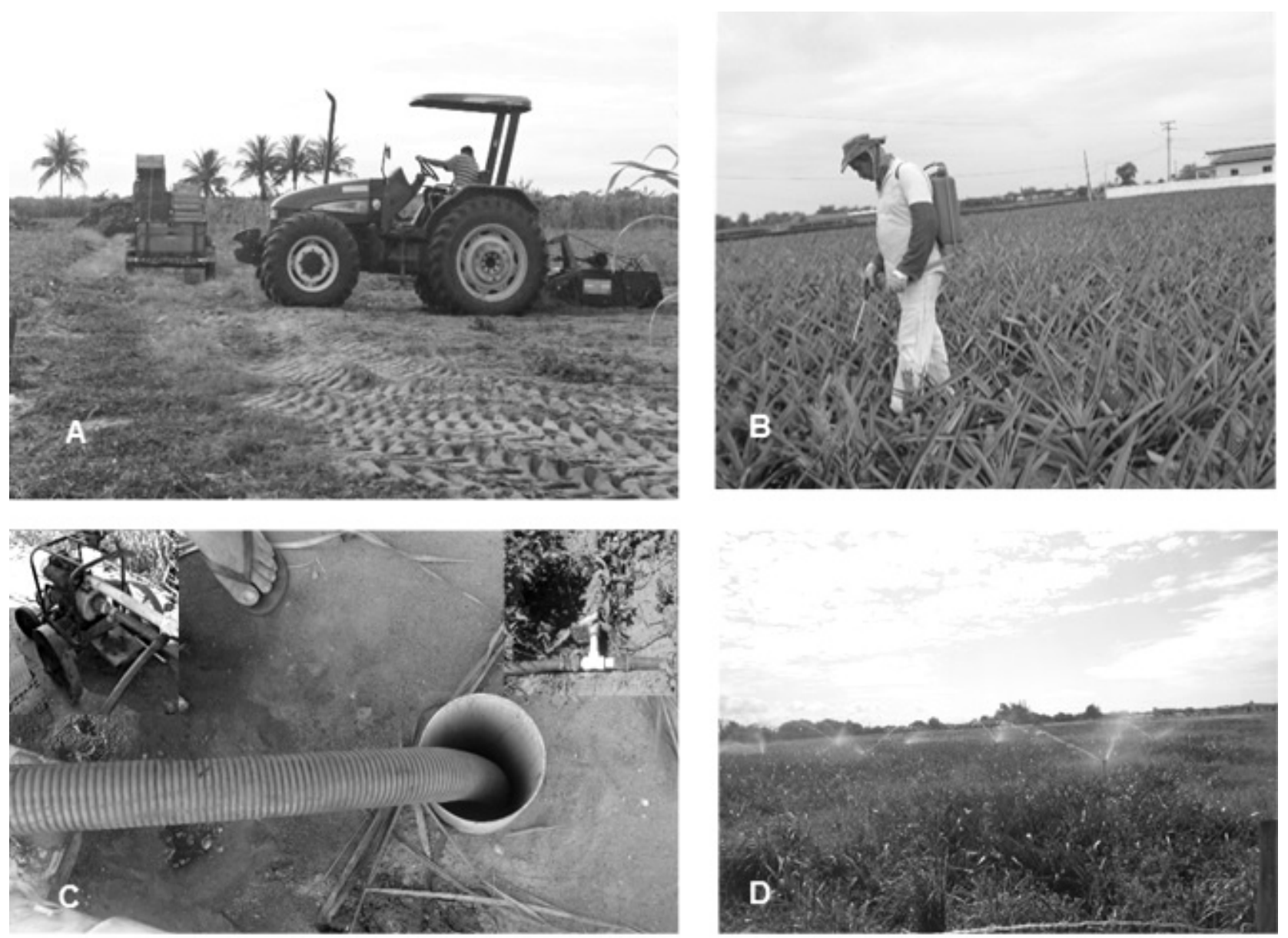

Fonte: Org. os autores. 
Agricultura familiar no norte do estado do Rio de Janeiro: identificação de manejo tradicional

Raquel da Silva Paes, Camilah Antunes Zappes

Apesar da utilização de algumas técnicas modernas, os agricultores utilizam ainda técnicas organizadas por eles e que desta maneira se inserem no manejo tradicional. Dentre os entrevistados, $58 \%$ $(n=29)$ utiliza uma mistura entre adubo químico e orgânico (excrementos de animais); 22\% (n=11) utiliza somente adubo químico; $18 \%(\mathrm{n}=9)$ usa apenas adubo orgânico e $2 \%(n=1)$ utiliza lodo de cana de açúcar. Ainda, são utilizadas técnicas de conservação do solo por meio da rotação de culturas $(\mathrm{n}=38 ; 76 \%)$ e pousio $(n=32 ; 64 \%)$. O pousio envolve o descanso total do solo em um período entre seis e 12 meses, momento em que nenhuma lavoura é plantada no terreno. Já a rotação de culturas é a alternância de plantio de espécies diferentes em um único terreno o que garante a inserção de nutrientes variados no solo. Estas etapas possibilitam a conservação do solo por meio de observação dos seus ciclos naturais, em que a rotação de culturas insere novos nutrientes no solo à medida que há variação nas espécies cultivadas e no seu descanso total na etapa de pousio. A figura 3 demonstra os níveis em que o manejo tradicional é realizado na prática da agricultura familiar no $5^{\circ}$ Distrito do município de São João da Barra, no norte fluminense.

Figura 3: Etapas do manejo tradicional praticado na agricultura familiar no município de São João da Barra, norte do estado do Rio de Janeiro.

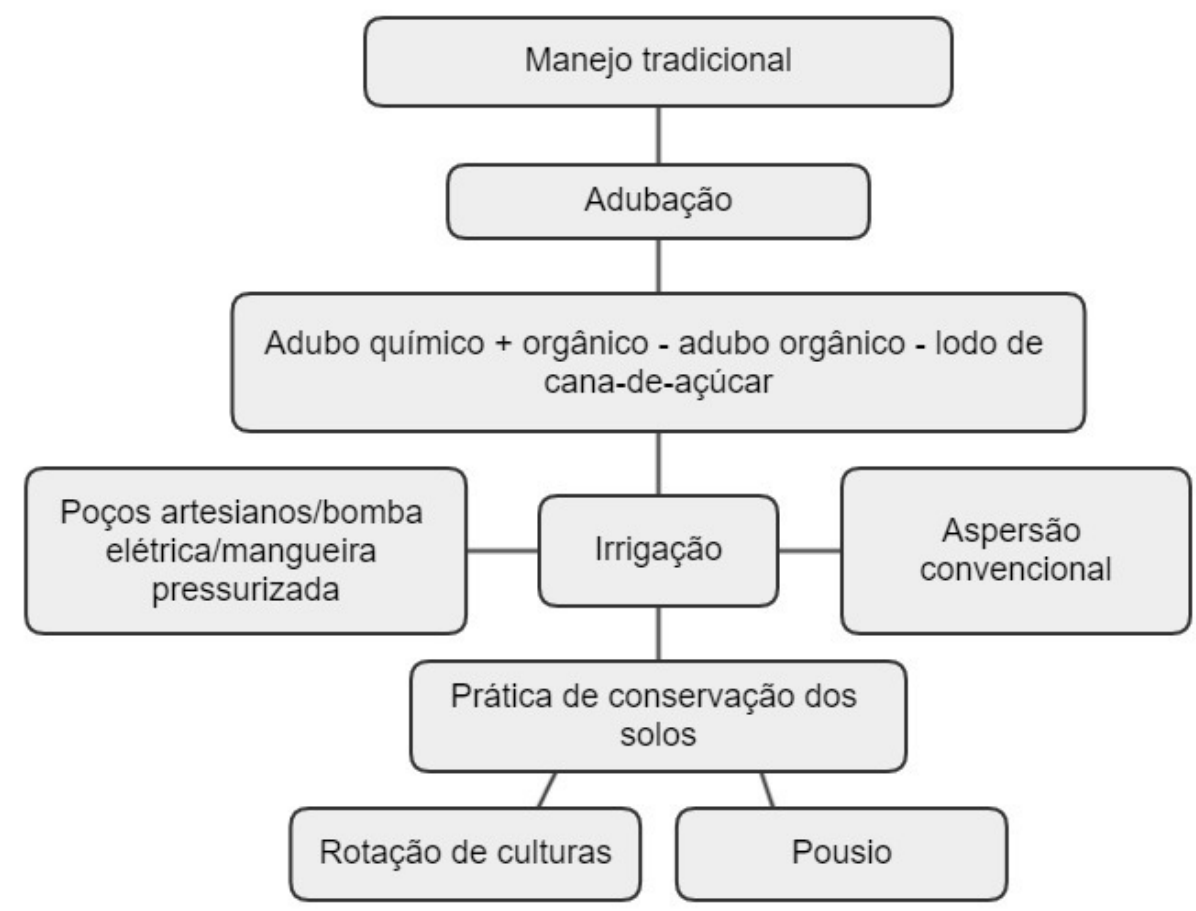

Fonte: Org. os autores.

\section{DISCUSSÃO}

Os agricultores familiares das comunidades pesquisadas no $5^{\circ}$ Distrito do município de São João da Barra são em sua maioria do sexo masculino com baixa escolaridade. Estas são características sociais comuns relacionadas ao perfil dos trabalhadores deste ramo econômico no país (CAMARANO et al., 1998; NEY et al., 2009; SOUZA, 2015). A educação é um fator que influencia diretamente no acesso de geração de renda e no processo de formação cidadã. Assim, a baixa escolaridade de populações rurais tem historicamente dificultado a sua organização social (SOUZA et al., 2015).

Populações rurais que desenvolvem a agricultura garantem sua sobrevivência a partir da atividade (ABRAMOVAY, 1997). Nas comunidades do $5^{\circ}$ Distrito do município de São João da Barra, a prática da agricultura familiar é inserida precocemente na vida dos agriculto- 
res, sendo repassada principalmente no núcleo familiar durante gerações o que demonstra ser esta uma atividade tradicional. Essa característica de reprodução ancestral da prática agrícola é um aspecto comum no país sendo repassado geralmente de pai para filho e desta maneira é estabelecida a tradição (ABRAMOVAY, 1997).

Os agricultores descreveram como principais espécies cultivadas o abacaxi, o quiabo e o maxixe, o que corrobora com estudos sobre o tema realizados anteriormente na região (BURLA et al., 2015). Segundo os entrevistados, as espécies cultivadas se adaptaram às condições de altas temperaturas e luminosidade $\mathrm{e}$ solos pobres em nutrientes o que demonstra que o saber tradicional agrícola envolve conhecimentos sobre as dinâmicas físicas do solo e as condições adequadas para o desenvolvimento dos plantios (MATUK, 2012).

Os agricultores familiares do $5^{\circ}$ Distrito do município de São João da Barra utilizam instrumentos que se dividem desde ferramentas manuais até maquinário pesado como tratores. Guanziroli et al. (2001) relataram que a agricultura familiar depende de forma intensa da força de trabalho com o uso de instrumentos manuais nos cultivos e com pequena inserção tecnológica. Entretanto a frequente utilização de tratores nas localidades estudadas provavelmente ocorre devido à disponibilidade do maquinário fornecido por projeto municipal social e empréstimo via associação de agricultores. Tal projeto é uma ação governamental que visa apoiar o pequeno produtor com apoio técnico e incentivo a venda dos produtos em feiras locais (PASSOS, 2013).

Dentre as técnicas e etapas utilizadas na agricultura familiar no $5^{\circ}$ Distrito do município de São João da Barra, o pousio e a rotação de culturas interferem diretamente na conservação do solo e por isso são usadas tradicionalmente há gerações. Os processos desta atividade descritos pelos agricultores também são registradas por Burla et al. (2015) que caracterizam a atividade como tradicional. A utilização de adubação química foi justificada pela ocorrência de déficit hídrico e consequentemente déficit nutricional do solo da região. Segundo os agricultores, quando há falta de água o solo torna-se pobre em nutrientes em que a alternativa para solucionar este problema é intensificar a adubação. $\mathrm{O}$ incremento de substâncias químicas ao solo sem orientação e observação técnica pode causar desequilíbrios nutricionais e prejuízo nas produções (SILVA et al., 2007; BURLA et al., 2015). Uma solução aos danos causados ao solo pelo déficit hídrico e nutricional é apresentada por Almeida et al. (2004) em que descrevem o uso de um modelo de irrigação por água subterrânea realizado no município de Pesqueira, região do semiárido do estado de Pernambuco, já que esta região apresenta baixos níveis de precipitação.

Neste estudo, o sistema de irrigação por poços artesianos com bomba elétrica e mangueira pressurizada se mostrou o mais frequentemente usado na região. Esta técnica é caracterizada como tradicional, pois se associa às condições naturais da área e é utilizada na forma de "cacimbas" (modelo que precedeu os poços artesianos) nos sistemas agrícolas desde antes das alterações de drenagem iniciadas no final do século XVII (CARNEIRO, 2004). Em sistemas lagunares da planície fluvio-marinha regional, como exemplo o norte fluminense, a disponibilidade de água permite o desenvolvimento de atividades tradicionais voltadas aos recursos aquáticos como a pesca artesanal e a agricultura familiar (BURLA et al., 2015). Atualmente, a irrigação de lavouras no $5^{\circ}$ Distrito do município de São João da Barra ainda está vinculada às características hídricas regionais, já que é mais intenso o uso de poços artesianos em detrimento ao modelo de aspersão convencional.

O modelo de irrigação de aspersão convencional é utilizado em menor quantidade no norte fluminense devido ao elevado investimento financeiro para montagem de sua estrutura. A aplicação deste modelo de irrigação tem sido apresentada em sistemas produtivos familiares de assentados no semiárido nordestino a partir de confecção artesanal o que reduz o seu custo (COELHO et al., 2012). Nesta mesma região do nordeste são utilizadas formas de irrigação por canos ou mangueiras perfuradas que se assemelham às utilizadas no $5^{\circ}$ Distrito do município de São João da Barra como auxílio ao modelo de irrigação por poços artesianos.

O uso de resíduos do processo de industrialização do açúcar na agricultura familiar no norte fluminense pode ter auxiliado no incremento da fertilidade dos solos que na área é baixa. Entretanto, atualmente a utilização deste resíduo é pequeno, provavelmente porque na região a indústria sulcroalcooleira está diminuindo sua atuação. A adubação química e orgânica descrita pelos agricultores foi a solução encontrada por eles para enriquecer 
o solo de nutrientes. Esta técnica tem sido utilizada no município de Coimbra, estado de Minas Gerais e tem apresentado resultados consistentes para a produtividade de grãos em lavouras de milho (SILVA et al., 2007).

As técnicas de pousio e rotação de culturas foram relatadas como as que atuam em conjunto com a adubação e irrigação como estratégia de conservação dos solos. De acordo com Boserup (1987) a técnica de pousio é classificada como longo/florestal, arbustivo, curto, anual e múltiplo de acordo com o desenvolvimento da vegetação natural em que não há presença de lavoura. No $5^{\circ}$ Distrito do município de São João da Barra é desenvolvido o pousio curto em que a vegetação apresenta características de ambientes campestres de pequeno porte, uso de adubação adicional de origem orgânica (excrementos de animais), e utilização de instrumentos manuais como arados e enxadas.

Em relação à rotação de culturas ocorre uma diversificação dos cultivos o que melhora o rendimento dos solos. Segundo os agricultores as lavouras são melhor desenvolvidas depois da alternância das espécies cultivadas. Nesse contexto, estudos sobre a funcionalidade dessa técnica a descrevem como importante na diminuição de doenças, e no equilíbrio da ciclagem de nutrientes nos solos, pois as espécies cultivadas inserem novos nutrientes nesse recurso o que consequentemente melhora o rendimento agrícola (WUTKE et al., 2000).

A partir do conhecimento tradicional de agricultores familiares foi possível compreender o modo como a atividade é realizada no $5^{\circ}$ Distrito do município de São João da Barra em que são utilizadas um conjunto de técnicas modernas e tradicionais, além de ter sido identificada a existência de manejo tradicional nas lavouras. Tal manejo foi desenvolvido ao longo de anos de observação do ambiente e o modo como as espécies cultivadas se desenvolviam em um solo com baixa fertilidade, altas temperaturas e grande luminosidade. $\mathrm{O}$ uso dos recursos naturais baseado nas experiências tradicionais geralmente está associado à relação ancestral entre comunidades e ambiente (HANAZAKI, 2003). Desta maneira, este conhecimento empírico pode ser utilizado na implementação e manutenção de políticas públicas direcionadas aos recursos naturais a serem conservados e manejados para a continuidade das atividades tradicionais como exemplo, a agricultura familiar no norte fluminense.

\section{CONCLUSÃO}

Os agricultores familiares do $5^{\circ}$ Distrito do município de São João da Barra são principalmente do sexo masculino com baixa escolaridade. Esta característica pode dificultar a organização dos envolvidos como um grupo social de geração de renda a partir de bens alimentícios comercializáveis. A propriedade rural é descrita por simbologismos e o conhecimento sobre a agricultura familiar foi desenvolvido ao longo de anos por meio da prática diária da atividade e repassado oralmente dentro do núcleo familiar principalmente.

O manejo do solo e técnicas utilizadas na produção agrícola na região se apresentam de forma variada e adaptada ao ambiente local destacando a produção de espécies como quiabo, maxixe e abacaxi, além de vegetais desenvolvidos em pequena escala como couve e alface. As principais formas de irrigação agrícola envolvem a irrigação por poços artesianos/bomba elétrica/ mangueira pressurizada seguido da aspersão convencional utilizada em menor frequência devido ao custo das instalações mecânicas. Devido à baixa fertilidade do solo são utilizados adubo químico, orgânico e lodo de cana de açúcar. Ainda, ocorrem políticas públicas voltadas à disponibilidade de maquinário pesado em projeto de incentivo ao pequeno produtor rural o que consequentemente auxilia na agricultura familiar local.

As técnicas de pousio e rotação de culturas junto à adubação mista e irrigação são utilizadas como práticas de manejo tradicional para a conservação do solo. A compreensão da percepção de agricultores sobre as técnicas e etapas de produções da agricultura familiar pode se tornar importante para o estabelecimento de políticas públicas. A identificação de ocorrência de manejo tradicional e a aproximação entre pesquisadores e comunidades agrícolas pode ser um fator facilitador de implementação e incremento de novas técnicas a fim de aumentar a produção na agricultura familiar e auxiliar na manutenção da atividade tradicional na região.

\section{AGRADECIMENTOS}

A todos os agricultores e funcionários da Prefeitura de São João da Barra, estado do Rio de Janeiro pela colaboração durante as coletas dos dados. A primeira autora agradece a CAPES pelo fomento 
Agricultura familiar no norte do estado do Rio de Janeiro: identificação de manejo tradicional Raquel da Silva Paes, Camilah Antunes Zappes

por meio de bolsa de pós-graduação. A segunda autora agradece à FAPERJ (E-26/203.202/2016) pelo fomento à pesquisa.

\section{REFERÊNCIAS}

ABRAMOVAY, R. Agricultura familiar e uso do solo. São Paulo em perspectiva. São Paulo, v. 11, n. 2, p. 73-78, 1997.

ALMEIDA, T. A.; NETTO, M. L. C.; MONTENEGRO, S. M. G. L.; et al. Utilização de águas subterrâneas em aluviões no Agreste pernambucano. Águas Subterrâneas. São Paulo, v. 28, 2014. Disponível em: $<\mathrm{http}$ ://aguassubterraneas.abas.org/asubterraneas/ article/view/23661/15737>. Acesso em: 30 abr. 2016. ALTAFIN, I. Reflexões sobre o conceito de agricultura familiar. Brasília: CDS/UnB, 2007.

ALTIERI, M. A.; TOLEDO, V. M. Natural resource management among small-scale farmers in semi-arid lands: Building on traditional knowledge and agroecology. Annals of Arid zone, v. 44, n. 3/4, p. 365, 2005.

BAILEY, K. D. Methods of social research. 2 ed. New York: McMillan Publishers. The Free Press. 1982.

BOSERUP, E. Evolução agrária e pressão demográfica. São Paulo: HUCITEC, 1987.

BURLA, R. S.; OLIVEIRA, V. P. S.; MANHÃES, C. M. C.; et al. Caracterização dos aspectos socioeconômicos e do processo produtivo agrícola dos produtores rurais da microbacia do Rio Doce, São João da Barra, RJ. Vértices. Campos dos Goytacazes, v. 17, n. 1, p. 149-162, 2015. Disponível em: <http://essentiaeditora.iff.edu.br/index.php/vertices/ article/view/18092667.20150010>. Acesso em: 10 nov. 2015. DOI: https://doi.org/10.5935/1809-2667.20150010.

BRASIL. Instituto Brasileiro de Geografia e Estatística. Município de São João da Barra. Disponível em: <http://www.cidades.ibge.gov.br/painel/painel. php?lang $=\&$ codmun $=330500 \&$ search $=\% 7$ Csao-joao-da-barra>. Acesso em: 06 mar. 2016.
BRASIL. Decreto No 11.326, de 24 de julho de 2006. Estabelece as diretrizes para formulação da Política Nacional da Agricultura Familiar e Empreendimentos Familiares Rurais. Diário Oficial da República Federativa do Brasil. Brasília, DF, 2006. Disponível em: < http://www.planalto.gov.br/ccivil_03/_ato20042006/2006/lei/111326.htm>. Acesso em: 16 mar. 2016.

CARNEIRO, Paulo Roberto Ferreira. Dos pântanos à escassez: uso da água e conflito na Baixada dos Goytacazes. Campos dos Goytacazes: Annablume, 2004.

COELHO, E. F.; SILVA, T. S. M.; SILVA, A. J. P.; et al. Sistemas de irrigação de baixo custo para agricultura familiar de assentamentos ribeirinhos do semiárido. In: GHEYI, H. R.; PAZ, V. P. S.; MEDEIROS, S. S.; et al. Recursos hídricos em regiões semiáridas: Estudos e aplicações. Campina Grande: Instituto Nacional do Semiárido; Cruz das Almas: Universidade Federal do Recôncavo da Bahia, 2012.

COUTINHO, R. R. Avaliação das transformações socioambientais oriundas da implantação do complexo portuário industrial do Açu. 2009. Dissertação (Mestrado) - Programa de Pós-Graduação em Engenharia Ambiental. Instituto Federal Fluminense (Campus Campos-Centro), Campos dos Goytacazes, 2009. Disponível em: $<$ http://www.dominiopublico.gov.br /pesquisa/DetalheObraForm.do? select_action $=\&$ co $_{-}$ obra $=168692>$. Acesso em: 08 mar. 2016.

COSTA NETO, E. M.; MARQUES, J. G. W. A etnotaxonomia de recursos ictiofaunísticos pelos pescadores da comunidade de Siribinha, Norte do Estado da Bahia, Brasil. Biociências. Taubaté, v. 8, n. 2, p. 61-76, 2000.

CHRISTENSEN, N. L.; BARTUSKA, A. M.; BROWN, J. H.; et al. The report of the ecological society of America Committee on the scientific basis for ecosystem management. Ecological Applications. Washington, v. 6, n. 3, p. 665-691, ago./1996. DOI: https://doi.org/10.2307/2269460.

DIEGUES, A. C. Etnoconservação da natureza: enfoques alternativos. In: DIEGUES, A. C. (Org.). Etnoconservação: novos rumos para a proteção da natureza nos trópicos. São Paulo: Hucitec, 2000. p. 1-46. 
Agricultura familiar no norte do estado do Rio de Janeiro: identificação de manejo tradicional

Raquel da Silva Paes, Camilah Antunes Zappes

GALIZONI, F. M.; RIBEIRO, E. M.; JÚNIOR, E. C. L.; et al. Agricultura familiar, água e estratégias produtivas no rio dos Cochos, campos gerais de Minas. In: Congresso Brasileiro de Economia, Administracao e Sociologia Rural 46, Rio Branco, AC, 2008. Anais... Rio Branco, AC, 2008. Disponível em: $<\mathrm{http}: / / w w w$. sober.org.br/palestra/9/553.pdf $>$. Acesso em: 06 mai. 2016.

GUANZIROLI, C. E.; CARDIM, S. E. C. S. (Coord.). O novo retrato da agricultura familiar: o Brasil redescoberto. Brasília: Projeto de cooperação técnica INCRA/FAO, 2000. Disponível em: <https://www. agencia.cnptia.embrapa.br/recursos/novoretratoID-3iTs4E7R59.pdf $>$. Acesso em: 02 abr. 2016.

GELCICH, S.; EDWARDS-JONES, G.; KAISER, M. J.; et al. Co-management policy can reduce resilience in traditionally managed marine ecosystems. Ecosystems, v. 9, n. 6, p. 951-966, 2006. DOI: https://doi. org/10.1007/s10021-005-0007-8.

HANAZAKI, N. Comunidades, conservação e manejo: o papel do conhecimento ecológico local. Biotemas. Florianópolis, v. 16, n. 1, p. 23-47, 2003.

KENDALL, L. The conduct of qualitative interviews: Research, Questions, Methodological Issues, and Researching Online. In: COIRO, J.; KNOBEL, M.; LANKSHEAR, C.; et al.. Handbook of research on new literacies. Taylor \& Francis, New York, USA. New York, USA, p. 133-149, 2008.

MALINOWSKI, B. Argonautas do Pacifico ocidental: um relato do empreendimento e da aventura dos nativos nos arquipélagos da Nova Guiné Melanésia. São Paulo: Abril, 1976.

MATUK, F. A. V. Território, conhecimento local e uso do solo na comunidade quilombola de Malhada Grande - norte de minas gerais. 2012. 147f. Dissertação (Mestrado) - Programa de Pós-Graduação em Solos e Nutrição de Plantas. Universidade Federal de Viçosa, Viçosa, 2012. Disponível em: $<$ http://www.locus.ufv. $\mathrm{br} / \mathrm{bitstream} / \mathrm{handle} / 123456789 / 5500 /$ texto\% $20 \mathrm{com}$ pleto.pdf?sequence=1>. Acesso em: 15 mai. 2016.
MELLO, L. G. Antropologia cultural, objetivo e método. In: MELLO, Luiz Gonzaga. Antropologia Cultural: Iniciação, teoria e temas. 11 ed. Petrópolis: Editora Vozes, 2004.

MIRANDA, T. M., HANAZAKI, N. Etnobotânica e Antropologia: descobertas, questionamentos e dificuldades em uma pesquisa de campo. In: Araújo, T. A. S.; Albuquerque, U. P. (Org.). Encontros e desencontros na pesquisa etnobiológica e etnoecológica: os desafios do trabalho em campo. Recife: NUPEEA. p.121-143.

MOURA, M. Â. T. Comparativo entre agricultura familiar teórica e normativa no Brasil. 2011. Monografia (Graduação) - Curso de Tecnólogo de Planejamento e Gestão para o Desenvolvimento Rural da faculdade de ciências econômicas. Universidade Federal do Rio Grande do Sul, Porto Alegre, 2011.

NETTING, R. Mc. Smallholders, householders: Farm families and the ecology of intensive, sustainable agriculture. Stanford: Stanford University Press, 1993. DOI: https://doi.org/10.2307/2804419.

PASSOS, W. S. Amargo presente da cana, doce passado do açúcar: a sobrevivência do discurso canavieiro nos programas de governo dos candidatos à prefeitura de Campos dos Goytacazes nas eleições de 2012. Anais: Encontros Nacionais da ANPUR. Rio de Janeiro, v. 15, 2013. Disponível em: $<$ http://unuhospedagem.com.br/revista/rbeur/index.php/anais/ article/ view/4364>. Acesso em: 30 abr. 2016.

PECH, J. A. N.; RIVERA, J. A. O.; DÍAZ, F. J. R.; et al. Diversidad y condiciones socioculturales de los solares mayas del municipio de Lázaro Cárdenas, Quintana Roo. In: CHÁVEZ-SERVIA, J. L.; ARIAS-REYES, L. M.; JARVIS, D. I.; et al. Manejo de la diversidad de los cultivos en los agroecosistemas tradicionales - Resúmenes del simposio: Manejo de la diversidade cultivada en los agroecosistemas tradicionales. Mérida: Instituto Internacional de Recursos Fitogenéticos, Roma, Itália. 2004. p. 62-63.

PIRES, B. O. Transformação produtiva de espaços rurais e seus impactos sobre populações locais: uma 
Agricultura familiar no norte do estado do Rio de Janeiro: identificação de manejo tradicional Raquel da Silva Paes, Camilah Antunes Zappes

análise da construção do Complexo Portuário Industrial do Açu no munícipio de São João da Barra, RJ. 2009. 111f. Dissertação (Mestrado) - Programa de Pós-Graduação em Políticas Sociais. Universidade Estadual do Norte Fluminense, Campos dos Goytacazes, 2009. Disponível em: $<$ http://uenf.br/pos-graduacao/ politicas-sociais/files/2015/06/BEATRIZ -DE-OLIVEIRA-PIRES.pdf $>$. Acesso em: 10 nov. 2015.

POSEY, D. A. Manejo da floresta secundária, capoeiras, campos e cerrados (kaiapó). In: RIBEIRO, Darcy (org.). Suma etnológica brasileira. 2. ed. Petropólis: Editora Vozes, 1987. p. 173-185

RIMA. Relatório de Impacto Ambiental. Infraestruturas do Distrito Industrial de São João da Barra. São João da Barra: Ecologus, 2011. Disponível em: $<$ http:// ceivap.org.br/downloads/ eia-rima-distrito-industrial-de-sao-joao-da-barra.pdf>. Acesso em: 07 out. 2016.

RYAN, G. W.; BERNARD, H. R. Data management and analysis methods. 2000.

SANCHES, R. A. Caiçaras e a estação ecológica de Jureia-Itatins: litoral sul de São Paulo. São Paulo: Annablume, 2004.

SILVA, T. O.; MENEZES, R. S. C. Adubação orgânica da batata com esterco e, ou, Crotalaria juncea. Revista Brasileira de Ciência do Solo. Viçosa, v. 31, n. 1, p. 51-61, 2007. DOI: https://doi.org/10.1590/s010006832007000100006 .

SOUZA, H. M.; NEY, M. G.; SOUZA, P. M.; et al. Escolaridade, carteira de trabalho e renda dos empregados no meio rural brasileiro. Campo-Território: Revista de Geografia Agrária. Uberlândia, v. 10, n. 20, p. 468-492, jul./2015. Disponível em: <http:// www.seer.ufu. br/index.php/campoterritorio/article/ view/27044/17032>. Acesso em: 30 mai. 2016.

SOUZA, H. N. Sistematização da experiência participativa com sistemas agroflorestais: rumo à sustentabilidade da agricultura familiar na Zona da Mata mineira. 2006. 145f. Tese (Doutorado) - Programa de Pós Graduação em Solos e Nutrição de Plantas. Universidade Federal de Viçosa, Viçosa, 2006.
SOUZA, T. N.; OLIVEIRA, V. P. S. Conflito socioambiental entre atividades de pesca artesanal marinha e implantação de atividades portuárias no Norte Fluminense. Boletim do Observatório Ambiental Alberto Ribeiro Lamego. Campos dos Goytacazes, v. 4, n. 2 , p. 219-230, 2010. DOI: https://doi.org/10.5935/21774560.20100021. Disponível em: <http://www. essentiaeditora.iff.edu.br/index.php/boletim/article/ view/2177-4560.20100021>. Acesso em: 19 jan. 2016.

WANDERLEY, M. N. B. Raízes históricas do campesinato brasileiro. In: TEDESCO, J. C. Agricultura familiar: realidades e perspectivas. Passo Fundo: EDIUPF, 1999.

WUTKE, E. B. et al. Propriedades do solo e sistema radicular do feijoeiro irrigado em rotação de culturas. Revista Brasileira de Ciência do Solo. Viçosa, v. 24, n. 3, p. 621-633, 2000. DOI: https://doi. org/10.1590/s0100-06832000000300015. 